\title{
CP, T and CPT tests with neutral kaons at KLOE-2
}

\section{Michał Silarski* on behalf of the KLOE-2 Collaboration}

Faculty of Physics, Astronomy and Applied Computer Science, Jagiellonian University, 30-348

Kraków, Poland

E-mail: michal.silarski@uj.edu.pl

We report on the recent results on $C P, T$ and $C P T$ tests in the neutral kaon sector obtained with the KLOE dataset. Search for the $C P$-violating decay $K_{S} \rightarrow 3 \pi^{0}$ is described. We present also recent measurement of the charge asymmetry for the $K_{S} \rightarrow \pi e v$ decay and preliminary results of direct test of $T$ and $C P T$ in neutral kaon transitions. Prospects of further improvement of these tests with KLOE-2 dataset are also given.

XIV International Conference on Heavy Quarks and Leptons (HQL2018)

May 27- June 1, 2018

Yamagata Terrsa, Yamagata,Japan

${ }^{*}$ Speaker. 


\section{Introduction}

The discrete symmetries appeared to be of fundamental importance in modern physics, especially in the description of fundamental interactions. In particle physics a special role is played by the discrete symmetries of parity operation $(P)$, charge conjugation, reversal in time $(T)$ and their combinations: $C P$ and $C P T$. Violation of the $P, C$ and $C P$ symmetries by the weak interaction was well established in the last century. The $C P$ non-conservation was initially discovered in the neutral kaon system via the decay of $K_{L}$ to two pion state [1] but there are other meson systems in which one can observe breaking of this symmetry. In recent years $C P$ violation was observed in $B$ meson decays, which appeared to be even stronger than for neutral kaons [2]. It was found in the $B^{0}-\bar{B}^{0}$ mixing [3,4] and directly in the decay amplitudes of $B^{0}[5,6], B^{+}[7-9]$ and $B_{s}^{0}[10]$. The direct $C P$ violation was also observed in the kaon system, even earlier than in the beauty sector [11]. Based on the Standard Model, one expects $C P$ symmetry breaking also in the $D$ meson system but it was not seen yet experimentally [2]. The weak interaction violates also the time reversal symmetry. Again, it has been observed only in neutral kaons (for $C P$-conjugate states) [12] and $B$ decays [13].

In view of the matter-antimatter unbalance in the universe, many experiments search for new sources of discrete symmetries violation, for example in baryonic systems [14] or in the leptonic sector, in particular in positronium decays [15-17] and neutrino oscillations [18].

Despite a big experimental effort made up to now, there are still several open issues in the neutral kaon system which need experimental data. A large $C P$ violation is expected in the rare $K_{L} \rightarrow \pi^{0} v v$ and $K^{+} \rightarrow \pi^{+} v v$ decays which recently have been searched for by the KOTO [19] and NA62 [20] experiments. $C P$ violation is still not well measured for the $K_{S}$ meson, especially in its three-pion and semileptonic decays. In this sector precise data was provided by the KLOE experiment which performed also several $C P T$ tests [21-27]. In this paper we present results of latest tests of $C P, T$ and $C P T$ made using the KLOE dataset and perspectives for further improvement using data taken recently by the KLOE-2 experiment.

\section{The KLOE experiment and its upgrade}

The KLOE experiment was operated at the DAФNE $e^{+} e^{-}$collider [28] working at a center of mass energy around the $\phi$ meson mass: $\sqrt{s}=1019.45 \mathrm{MeV}$. In the period 2000-2006 KLOE gathered integrated luminosity of about $2.5 \mathrm{fb}^{-1}$ at the $\phi$ peak and around $250 \mathrm{pb}^{-1}$ at $\sqrt{s}=1000 \mathrm{MeV}$ [21]. The KLOE detector is presented schematically in Fig. 1. It consists of two main parts, a large drift chamber [29] and 4- $\pi$ lead scintillator electromagnetic calorimeter [30]. The drift chamber, $4 \mathrm{~m}$ in diameter and $3.3 \mathrm{~m}$ long, was built out of 12582 drift cells with tungsten sense wires. It is constructed out of carbon fiber composite and uses a gas mixture of helium (90\%) and isobutane (10\%). The drift chamber provides three-dimensional tracking with resolution in the bending plane of about $200 \mu \mathrm{m}$, resolution on the z-coordinate measurement of about $2 \mathrm{~mm}$ and of $1 \mathrm{~mm}$ on the decay vertex position. Momentum of a particle is determined from the curvature of its trajectory in the magnetic field with a fractional accuracy $\sigma_{p} / p=0.4 \%$ for polar angles larger than $45^{\circ}$ [21]. The KLOE electromagnetic calorimeter covers $98 \%$ of the solid angle. It is composed in total by 88 modules forming a barrel and two side detectors. Each module is built out of $1 \mathrm{~mm}$ diameter scintillating fibers grouped in cells of $4.4 \times 4.4 \mathrm{~cm}^{2}$ and embedded in 0.5 


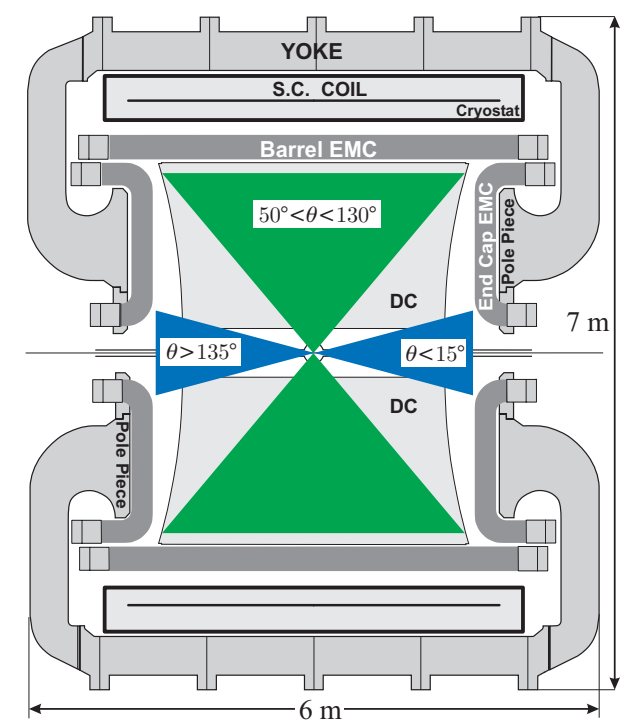

Figure 1: Schematic view of the KLOE detector.

$\mathrm{mm}$ lead foils, and it is read out from both sides by photomultipliers [31]. The KLOE calorimeter allows to measure particle energies and flight times with accuracies of $\sigma_{E} / E=5.7 \% / \sqrt{E[\mathrm{GeV}]}$ and $\sigma(t)=57 \mathrm{ps} / \sqrt{E[\mathrm{GeV}]} \oplus 100 \mathrm{ps}$, respectively [30]. It provides also the particle hit position with accuracy of about $1 \mathrm{~cm}$ in the plane transverse to the fibers direction while the longitudinal coordinate precision is energy dependent: $\sigma_{z}=1.2 \mathrm{~cm} / \sqrt{E[\mathrm{GeV}]}[21,32]$.

To complete and extend the KLOE physics program in 2014 the KLOE-2 Collaboration started a new data-taking campaign with an upgraded detector [33]. To improve vertex reconstruction capabilities KLOE was equipped with an inner tracker [34] near the interaction region. To register electrons and positrons from the $\gamma \gamma$ interaction with energy up to around $400 \mathrm{MeV}$ we have placed near the interaction point a small calorimeter. This Low Energy Tagger (LET) was built out of LYSO crystals read out by silicon photomultipliers [35]. The second detector supporting the $\gamma \gamma$ physics studies, High Energy Tagger (HET), was inserted inside the machine lattice about $11 \mathrm{~m}$ away from the KLOE interaction point providing measurement of the displacement of the scattered leptons with respect to the main orbit. This position-sensitive detector consists of 30 small BC418 scintillators $3 \times 3 \times 5 \mathrm{~mm}^{3}$, which provides spatial resolution of $2 \mathrm{~mm}$ (corresponding to momentum resolution of $\sim 1 \mathrm{MeV} / \mathrm{c}$ ) [36]. Two additional calorimeters, QCALT and CCALT, were installed to increase the KLOE acceptance. QCALT covers a region of DAФNE quadrupoles and it is composed of five layers of $5 \mathrm{~mm}$ thick scintillator plates alternated with $3.5 \mathrm{~mm}$ thick tungsten slabs, for a total depth of $4.75 \mathrm{~cm}$ [37]. The crystal calorimeter CCALT is placed near the interaction point to increase the KLOE acceptance in the low polar angle region down to $8^{\circ}$. The basic layout consists of two small barrels of LYSO crystals with SiPM readout [38].

The KLOE-2 data-taking campaign was concluded in 2018 with an integrated luminosity $L_{i n t}=$ $5.5 \mathrm{fb}^{-1}$ which, together with the old KLOE dataset, constitutes the biggest sample collected at the $\phi$ mass peak. Taking into account the entanglement of kaons originating from the $\phi$ meson decays and the possibility to tag one kaon with a decay or interaction of the other, KLOE-2 has a unique 
opportunity to test discrete symmetries in the kaon sector with a very high precision.

\section{Recent results on CP, T and CPT tests and perspectives for the KLOE-2}

The studies of the neutral kaon system with the KLOE data were focused in last years on measurement of the $C P$-violating $K_{S} \rightarrow 3 \pi$ decays [23] and tests of the $C P T, T$ and Lorentz symmetries via the determination of charge asymmetry for the $K_{S} \rightarrow \pi e v$ decay [39] and studies of the entanglement of the $K_{S}$ and $K_{L}$ states [24].

\subsection{Search for the $K_{S} \rightarrow 3 \pi$ decays}

$K_{S}$ decays into three-pion final states are still poorly known, the current value of the $K_{S} \rightarrow$ $\pi^{+} \pi^{-} \pi^{0}$ branching fraction amounts to $B R\left(K_{S} \rightarrow \pi^{+} \pi^{-} \pi^{0}\right)=\left(3.5_{-0.9}^{+1.1}\right) \cdot 10^{-7}$ [2], and the $K_{S} \rightarrow$ $3 \pi^{0}$ has never been observed. The $\left|\pi^{+} \pi^{-} \pi^{0}\right\rangle$ final state can be produced in neutral kaon decays with violation of the $C P$ symmetry (for isospin $I=1$ and $I=3$ ) and without $(I=0$ and $I=2$ ). The latter decays are strongly suppressed by the centrifugal barrier effects [2]. Anyhow, the two kinds of final states can be separated by the analysis of the $\pi^{+} \pi^{-} \pi^{0}$ Dalitz plot, thus allowing the determination of the $K_{S}$ to $K_{L}$ decay amplitude ratio $\eta_{+-0}=\frac{A\left(K_{S} \rightarrow \pi^{+} \pi^{-} \pi^{0}\right)}{A\left(K_{L} \rightarrow \pi^{+} \pi^{-} \pi^{0}\right)} \cong \varepsilon+\varepsilon_{+-0}^{\prime}$, where $\varepsilon$ indicates the $K_{S} C P$ impurity and $\varepsilon_{+-0}^{\prime}$ is the contribution of the direct $C P$-violating term [31]. In the case of $\left|\pi^{0} \pi^{0} \pi^{0}\right\rangle$ final state, only isospin $I=1$ or $I=3$ is allowed and the $K_{S} \rightarrow 3 \pi^{0}$ decay is a purely $C P$ violating process, for which one defines the analogous ratio: $\eta_{000}=\frac{A\left(K_{S} \rightarrow \pi^{0} \pi^{0} \pi^{0}\right)}{A\left(K_{L} \rightarrow \pi^{0} \pi^{0} \pi^{0}\right)} \cong \varepsilon+$ $\varepsilon_{000}^{\prime}$. As mentioned the $K_{S} \rightarrow 3 \pi^{0}$ decay was not yet observed and the Standard Model prediction of its branching fraction is about $2 \cdot 10^{-9}$ [40].

KLOE has searched for the $K_{S} \rightarrow 3 \pi^{0}$ and set the best upper limit on $\left|\eta_{000}\right|$ and branching ratio for this decay. The measurement was based on tagging the $K_{S}$ with the interaction of the $K_{L}$ meson in the KLOE calorimeter. The signal signature was composed then by six prompt $\gamma$ quanta originating from the interaction point. Background for this process was composed mainly by the $K_{S} \rightarrow 2 \pi^{0}$ with two reconstructed photons originating from shower splitting in the calorimeter or accidental coincidence with DA $\Phi$ NE background. After the whole analysis chain we have found no candidate events in the signal region for both data and Monte Carlo background simulation. This corresponds to the upper limit on the branching fraction $B R\left(K_{S} \rightarrow 3 \pi^{0}\right) \leq 2.6 \times 10^{-8}$ at $90 \%$ C.L. which can be translated into a limit on the amplitude ratio: $\left|\eta_{000}\right|=\left|\frac{A\left(K_{S} \rightarrow 3 \pi^{0}\right)}{A\left(K_{L} \rightarrow 3 \pi^{0}\right)}\right|=\sqrt{\frac{\tau_{L}}{\tau_{S}} \frac{B R\left(K_{S} \rightarrow 3 \pi^{0}\right)}{B R\left(K_{L} \rightarrow 3 \pi^{0}\right)}} \leq 0.0088$ at 90\% C.L. [23]. With the additional data collected with the KLOE-2 detector we expect to further improve the sensitivity of this search by the combined analysis of both datasets.

\subsection{Measurement of the charge asymmetry for the $K_{S} \rightarrow \pi e v$ decay}

Semileptonic neutral kaon decays provide tests of many fundamental aspects of the Standard Model, including the $C P$ and $C P T$ symmetries [27]. The charge asymmetries for $K_{S}$ and $K_{L}$ defined as:

$$
A_{S, L}=\frac{\Gamma\left(K_{S, L} \rightarrow \pi^{-} e^{+} v\right)-\Gamma\left(K_{S, L} \rightarrow \pi^{+} e^{-} \bar{v}\right)}{\Gamma\left(K_{S, L} \rightarrow \pi^{-} e^{+} v\right)+\Gamma\left(K_{S, L} \rightarrow \pi^{+} e^{-} \bar{v}\right)}
$$


are sensitive to $C P$ violation [39]. If $C P T$ symmetry is exact the two asymmetries are expected to be identical and equal to the degree of the $C P$ impurity in the kaon mixing [39]:

$$
A_{S}=A_{L}=2 \operatorname{Re}(\varepsilon) \simeq 3 \times 10^{-3} .
$$

The sum and difference of $A_{S}$ and $A_{L}$ can be parametrized as:

$$
\begin{gathered}
A_{S}+A_{L}=4[\operatorname{Re}(\varepsilon)-\operatorname{Re}(y)] \\
A_{S}-A_{L}=4\left[\operatorname{Re}(\delta)+\operatorname{Re}\left(x_{-}\right)\right],
\end{gathered}
$$

where non-zero values of $\delta, \operatorname{Re}(y)$ and $\operatorname{Re}\left(x_{-}\right)$correspond to $C P T$ violation in the kaon mass matrix, $\Delta S=\Delta Q$ and $\Delta S \neq \Delta Q$ decay amplitudes, respectively.

$A_{L}$ has been measured by the KTeV Collaboration with a good precision: $A_{L}=\left(3.322 \pm 0.058_{\text {stat }} \pm\right.$ $\left.0.047_{\text {syst }}\right) \times 10^{-3}$ [41]. The best measurement of its counterpart, $A_{S}$, has been performed by KLOE using $410 \mathrm{pb}^{-1}$ of integrated luminosity with an uncertainty dominated by the statistical uncertainty [27,39]. Recently, an analogous analysis was performed on the rest of the KLOE sample. Again the $K_{S}$ sample was obtained by tagging with the $K_{L}$ interaction in the calorimeter. The selection of $K_{S} \rightarrow \pi e v$ decays was then performed asking for events with reconstructed vertex formed by two tracks with opposite curvature close to the $e^{+} e^{-}$interaction point. Both reconstructed tracks had to be associated with clusters in the calorimeter, which allowed to identify the charged particles using the Time-Of-Flight technique. The main source of background originated from the $K_{S} \rightarrow \pi^{+} \pi^{-}$decays and charged kaons. The best separation between the signal and background components was obtained with the variable: $M^{2}(e)=\left(E_{K_{S}}-E_{\pi}-p_{m i s s}\right)^{2}-\vec{p}_{e}^{2}$, where $p_{\text {miss }}=\left|\vec{p}_{K_{S}}-\vec{p}_{e}-\vec{p}_{\pi}\right| . \vec{p}_{e}$ denotes the measured momentum of lepton and $E_{K_{S}}$ and $\vec{p}_{K_{S}}$ are the $K_{S}$ energy and momentum, respectively. Analogously $E_{\pi}$ and $\vec{p}_{\pi}$ stand for energy and momentum of the identified pion. For the signal events this variable peaks close to zero (see Figure 2) [39]. A data sample of $K_{L} \rightarrow \pi e v$ decay tagged by the $K_{S} \rightarrow \pi^{0} \pi^{0}$ was used as a control sample. The obtained $A_{S}$ value was combined with the older KLOE result yielding the following number [39]:

$$
A_{S}=\left(-3.8 \pm 5.0_{\text {stat }} \pm 2.6_{\text {syst }}\right) \times 10^{-3} \text {. }
$$

Taking into account the experimental estimations of $A_{S}$ and $A_{L}$ we have also computed values of the $C P T$ violation parameters [39]:

$$
\begin{gathered}
\operatorname{Re}\left(x_{-}\right)=(-2.0 \pm 1.4) \times 10^{-3}, \\
\operatorname{Re}(y)=(1.7 \pm 1.4) \times 10^{-3},
\end{gathered}
$$

As one can see, the dominant uncertainty of these results are still statistical and we expect further improvement with the additional KLOE-2 data.

\subsection{Direct test of $T$ and $C P T$ in neutral kaon transitions}

Besides the test of the time reversal symmetry done by the CPLEAR Collaboration for $C P$ conjugate neutral kaon states [12], one can perform direct test of $T$ and $C P T$ symmetries using 

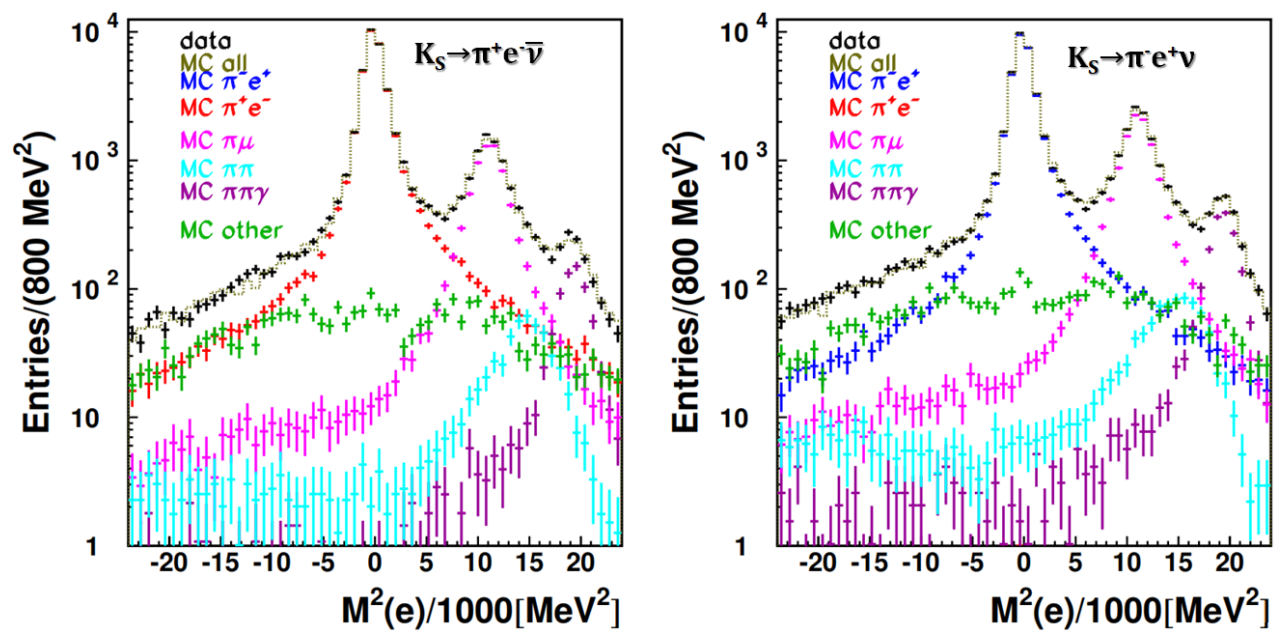

Figure 2: $M^{2}(e)$ distribution for data (black points) and fitted MC simulations (dotted histogram) for the two semileptonic $K_{S}$ decays used to construct $A_{S}$. The individual MC contributions are superimposed in the plots. Figure adapted from [39].

kaon transitions between their pure flavour $K, \bar{K}$ and $C P$-definite states $\left\{K_{+}, K_{-}\right\}$[42-44]. The strangeness states can be identified by semileptonic decays $K \rightarrow \pi^{-} e^{+} v$ and $\bar{K} \rightarrow \pi^{+} e^{-} \bar{v}$ while $K_{+}$and $K_{-}$should decay into two $\left(K_{+} \rightarrow \pi^{+} \pi^{-}\right)$and three pions $\left(K_{-} \rightarrow 3 \pi^{0}\right)$. The initial state of the kaon undergoing the transition is known from the decay of its quantum-entangled partner (see Fig. 3) [42]. One can define the following ratios of probabilities of mentioned transitions as a

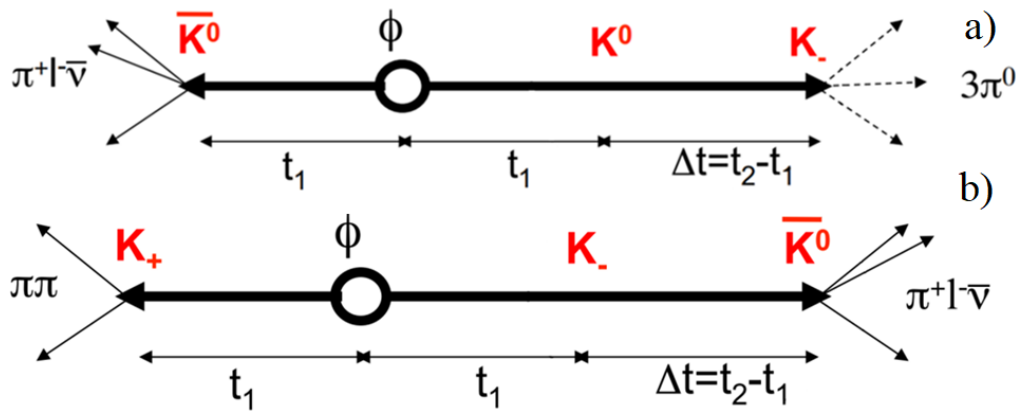

Figure 3: A scheme of the principle of the direct $C P T$ test in neutral kaon transitions. The $K^{0} \rightarrow K_{-}$ transition is identified by the semileptonic decay of $\bar{K}^{0}$ and the $3 \pi^{0}$ in the final state (a). The $C P T$ conjugate transition can be found by looking for the same semileptonic state accompanied by the $K_{+} \rightarrow \pi^{+} \pi^{-}$decay (b).

function of the decay time difference for the two kaons:

$$
\begin{aligned}
& R_{2, C P T}(\Delta t) \sim P\left(K \rightarrow K_{-}\right) / P\left(K_{-} \rightarrow \bar{K}\right) \\
& R_{4, C P T}(\Delta t) \sim P\left(\bar{K} \rightarrow K_{-}\right) / P\left(K_{-} \rightarrow K\right)
\end{aligned}
$$

The asymptotic values of $R_{2, C P T}$ and $R_{4, C P T}$ for $\Delta t \gg \tau_{S}$ (where $\tau_{S}$ denotes the $K_{S}$ lifetime) are sensitive to the $T$ violation, while their ratio is related to the $C P T$ violation parameters [44]:

$$
\frac{R_{2, C P T}\left(\Delta t \gg \tau_{S}\right)}{R_{4, C P T}\left(\Delta t \gg \tau_{S}\right)}=1-8 \operatorname{Re}(\delta)-8 \operatorname{Re}\left(x_{-}\right) .
$$




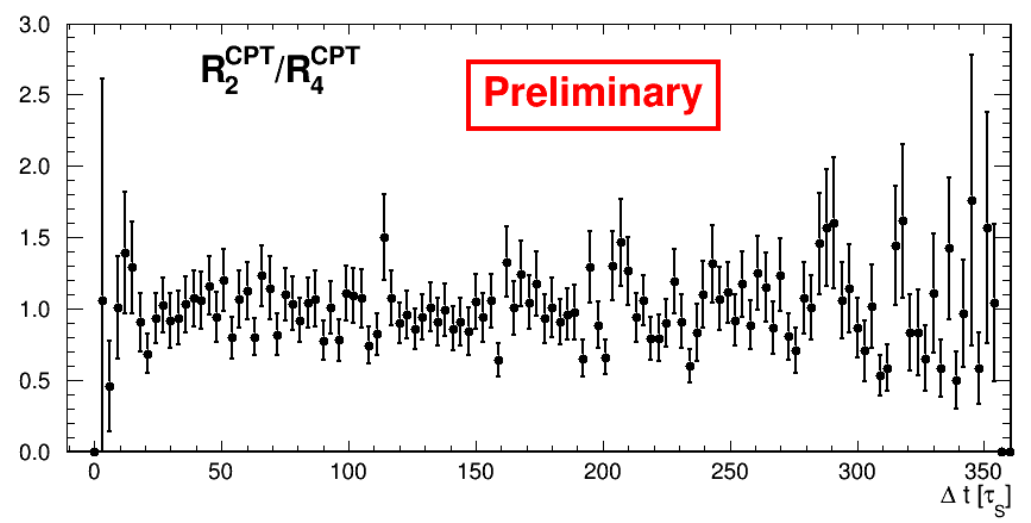

Figure 4: Preliminary distribution of the $C P T$-asymmetric double ratio $R_{2, C P T} / R_{4, C P T}$ obtained with the KLOE data. Figure adapted from [42].

Measurement of the $R_{2, C P T}$ and $R_{4, C P T}$ ratios has been performed on the full KLOE dataset. The analysis was performed for two classes of events. The first one with early kaon decay into a semileptonic final state with two tracks registered in the KLOE drift chamber, and a later kaon decay into $3 \pi^{0}$ resulting in up to $6 \gamma$ quanta [45]. The other class constituted events with an early kaon decay into two charged pions with two tracks originating close to the $\phi$ decay point, and a later semileptonic decay. Preliminary results of this analysis are presented in Fig. 4. With the KLOE-2 data sample we expect to reach a precision of about $10^{-3}$ on $R_{2, C P T} / R_{4, C P T}$.

\section{Summary}

Studies of the discrete symmetries are of great importance in view of understanding many fundamental issues of astrophysics and particle physics, including the asymmetry of matter and antimatter in the Universe. KLOE has significantly contributed to the understanding of the $C P$ violation in the kaon sector and provided many tests of other symmetries conservation, especially $C P T$. The new data gathered by the KLOE-2 Collaboration combined with the old dataset enables further improvement of the discrete symmetries studies in the neutral kaon sector.

\section{Acknowledgments}

We warmly thank our former KLOE colleagues for the access to the data collected during the KLOE data taking campaign. We thank the DAФNE team for their efforts in maintaining low background running conditions and their collaboration during all data taking. We want to thank our technical staff: G.F. Fortugno and F. Sborzacchi for their dedication in ensuring efficient operation of the KLOE computing facilities; M. Anelli for his continuous attention to the gas system and detector safety; A. Balla, M. Gatta, G. Corradi and G. Papalino for electronics maintenance; C. Piscitelli for his help during major maintenance periods. This work was supported in part by the Polish National Science Centre through the Grants No. 2013/11/B/ST2/04245, 2014/14/E/ST2/00262, 2014/12/S/ST2/00459, 2016/21/N/ST2/01727, 2016/23/N/ST2/01293, 2017/26/M/ST2/00697. 


\section{References}

[1] J. H. Christenson, J. W. Cronin, V. L. Fitch, R. Turlay Evidence for the $2 \pi$ Decay of the $K_{2}^{0}$ Meson, Phys. Rev. Lett. 13, (1964) 138.

[2] M. Tanabashi et al. (Particle Data Group) The Review of Particle Physics (2018) 233, Phys. Rev. D 98, (2018) 030001.

[3] B. Aubert et al. [BaBar Collaboration], Observation of CP violation in the $B^{0}$ meson system, Phys. Rev. Lett. 87 (2001) 091801 [hep-ex/0107013].

[4] K. Abe et al. [Belle Collaboration], Observation of large CP violation in the neutral B meson system, Phys. Rev. Lett. 87 (2001) 091802 [hep-ex/0107061].

[5] B. Aubert et al. [BaBar Collaboration], Observation of direct CP violation in $B^{0} \rightarrow K^{+} \pi^{-}$decays, Phys. Rev. Lett. 93 (2004) 131801 [hep-ex/0407057].

[6] Y. Chao et al. [Belle Collaboration], Evidence for direct CP violation in $B^{0} \rightarrow K^{+} \pi^{-}$decays, Phys. Rev. Lett. 93 (2004) 191802 [hep-ex/0408100].

[7] A. Poluektov et al. [Belle Collaboration], Evidence for direct CP violation in the decay $B \rightarrow D(*) K, D \rightarrow K_{S} \pi^{+} \pi^{-}$and measurement of the CKM phase $\phi_{3}$, Phys. Rev. D 81 (2010) 112002 [arXiv:1003.3360 [hep-ex]].

[8] P. del Amo Sanchez et al. [BaBar Collaboration], Measurement of CP observables in $B^{+-} \rightarrow D_{C P} K^{+-}$decays and constraints on the CKM angle $\gamma$, Phys. Rev. D 82 (2010) 072004 [arXiv:1007.0504 [hep-ex]].

[9] R. Aaij et al. [LHCb Collaboration], Observation of CP violation in $B^{ \pm} \rightarrow D K^{ \pm}$decays, Phys. Lett. B 712 (2012) 203, Erratum: [Phys. Lett. B 713 (2012) 351] [arXiv:1203.3662 [hep-ex]].

[10] R. Aaij et al. [LHCb Collaboration], First observation of CP violation in the decays of $B_{s}^{0}$ mesons, Phys. Rev. Lett. 110 (2013) 221601 [arXiv:1304.6173 [hep-ex]].

[11] A. Alavi-Harati et al. [KTeV Collaboration)], Observation of Direct CP Violation in $K_{S, L} \rightarrow \pi \pi$ Decays, Phys. Rev. Lett. 83 (1999) 22 [hep-ex/9905060].

[12] A. Angelopoulos et al. [CPLEAR Collaboration], First direct observation of time reversal noninvariance in the neutral kaon system, Phys. Lett. B 444 (1998) 43.

[13] J. P. Lees et al. [BaBar Collaboration], Observation of Time Reversal Violation in the $B^{0}$ Meson System, Phys. Rev. Lett. 109 (2012) 211801 [arXiv:1207.5832 [hep-ex]].

[14] R. Aaij et al. [LHCb Collaboration], Measurement of matter-antimatter differences in beauty baryon decays, Nature Phys. 13 (2017) 391 [arXiv:1609.05216 [hep-ex]].

[15] D. Kamińska et al. [J-PET Collaboration], A feasibility study of ortho-positronium decays measurement with the J-PET scanner based on plastic scintillators, Eur. Phys. J. C 76 (2016) 445 [arXiv:1607.08588 [physics.ins-det]].

[16] P. Moskal et al. [J-PET Collaboration], Potential of the J-PET detector for studies of discrete symmetries in decays of positronium atom - a purely leptonic system, Acta Phys. Polon. B 47 (2016) 509 [arXiv:1602.05226 [nucl-ex]].

[17] T. Yamazaki, T. Namba, S. Asai and T. Kobayashi, Search for CP violation in Positronium Decay, Phys. Rev. Lett. 104 (2010) 083401 Erratum: [Phys. Rev. Lett. 120 (2018), 239902] [arXiv:0912.0843 [hep-ex]]. 
[18] K. Abe et al. [T2K Collaboration], Measurement of neutrino and antineutrino oscillations by the T2K experiment including a new additional sample of $v_{e}$ interactions at the far detector, Phys. Rev. D 96 (2017) 092006 [arXiv:1707.01048 [hep-ex]].

[19] T. Yamanaka [KOTO Collaboration], The J-PARC KOTO experiment, Prog. Theor. Exp. Phys. (2012) 02 B006.

[20] M. Mirra [NA62 Collaboration], Results and prospects on kaon physics with the NA62 experiment at CERN, Nuovo Cimento C $\mathbf{0 3 8}$ (2015) 13.

[21] F. Bossi et al. [KLOE Collaboration], Precision Kaon and Hadron Physics with KLOE, Riv. Nuovo Cim. 31 (2008) 531 [arXiv:0811.1929 [hep-ex]].

[22] F. Ambrosino et al. [KLOE Collaboration], Determination of CP and CPT violation parameters in the neutral kaon system using the Bell-Steinberger relation and data from the KLOE experiment, JHEP 0612 (2006) 011 [hep-ex/0610034].

[23] D. Babusci et al. [KLOE Collaboration], A new limit on the CP violating decay $K_{S} \rightarrow 3 \pi^{0}$ with the KLOE experiment, Phys. Lett. B 723 (2013) 54 [arXiv:1301.7623 [hep-ex]].

[24] D. Babusci et al. [KLOE-2 Collaboration], Test of CPT and Lorentz symmetry in entangled neutral kaons with the KLOE experiment, Phys. Lett. B 730 (2014) 89 [arXiv:1312.6818 [hep-ex]].

[25] F. Ambrosino et al. [KLOE Collaboration], First observation of quantum interference in the process $\phi \rightarrow K_{S} K_{L} \rightarrow \pi^{+} \pi^{-} \pi^{+} \pi^{-}:$A Test of quantum mechanics and CPT symmetry, Phys. Lett. B 642 (2006) 315 [hep-ex/0607027].

[26] A. Di Domenico et al. [KLOE Collaboration], Search for CPT violation and decoherence effects in the neutral kaon system, J.Phys.Conf.Ser. 171 (2009) 012008.

[27] F. Ambrosino et al. [KLOE Collaboration], Study of the branching ratio and charge asymmetry for the decay $K_{s} \rightarrow \pi e v$ with the KLOE detector, Phys. Lett. B 636 (2006) 173 [hep-ex/0601026].

[28] A. Gallo et al., DAFNE status report, Conf. Proc. C 060626 (2006) 604.

[29] M. Adinolfi et al. [KLOE Collaboration], The tracking detector of the KLOE experiment, Nucl. Instrum. Meth. A 488 (2002) 51.

[30] M. Adinolfi et al. [KLOE Collaboration], The KLOE electromagnetic calorimeter, Nucl. Instrum. Meth. A 482 (2002) 364.

[31] M. Silarski et al. [KLOE Collaboration], Search for the CP violating $K_{S} \rightarrow 3 \pi^{0}$ decay with the KLOE detector, J. Phys. Conf. Ser 447 (2013) 012028.

[32] M. Silarski et al. [KLOE-2 Collaboration], Physics topics at KLOE-2, Hyperfine Interact. 211 (2012) 33 [arXiv:1111.4149 [hep-ex]].

[33] G. Amelino-Camelia et al. [KLOE-2 Collaboration], Physics with the KLOE-2 experiment at the upgraded DAФNE, Eur. Phys. J. C 68 (2010) 619 [arXiv:1003.3868 [hep-ex]].

[34] A. Di Cicco and G. Morello et al. [KLOE-2 Collaboration], Commissioning of the Inner Tracker of the KLOE-2 experiment, Acta Phys. Polon. B 46 (2015) 73.

[35] D. Babusci et al. [KLOE-2 Collaboration], Commissioning of the new taggers of the KLOE-2 experiment, Acta Phys. Polon. B 46 (2015) 81.

[36] F. Archilli et al. [KLOE-2 Collaboration], GammaâĂŞgamma tagging system for KLOE2 experiment, Nucl. Instrum. Meth. A 617 (2010) 266. 
[37] M. Cordelli et al. [KLOE-2 Collaboration], QCALT: A Tile calorimeter for KLOE-2 experiment, Nucl. Instrum. Meth. A 617 (2010) 105 [arXiv:0906.1133 [physics.ins-det]].

[38] F. Happacher et al. [KLOE-2 Collaboration], Commissioning of the new calorimeters of the KLOE-2 experiment, Acta Phys. Polon. B 46 (2015) 87 [arXiv:1501.05442 [physics.ins-det]].

[39] A. Anastasi et al. [KLOE Collaboration], Measurement of the charge asymmetry for the $K_{S} \rightarrow \pi e v$ decay and test of CPT symmetry with the KLOE detector, Submitted to JHEP [arXiv:1806.08654 [hep-ex]].

[40] Maiani L and Paver N 1995 The Second DAФNE Physics Handbook ed Maiani L, Pancheri G and Paver N (Frascati: Frascati Phys. Ser.) p 51

[41] A. Alavi-Harati et al. [KTeV Collaboration], A Measurement of the $K(L)$ charge asymmetry, Phys. Rev. Lett. 88 (2002) 181601 [hep-ex/0202016].

[42] A. Gajos [KLOE-2 Collaboration], Tests of discrete symmetries and quantum coherence with neutral kaons at the KLOE-2 experiment, Acta Phys. Polon. B 48 (2017) 1975 [arXiv:1710.08197 [hep-ex]].

[43] J. Bernabeu, A. Di Domenico and P. Villanueva-Perez, Direct test of time-reversal symmetry in the entangled neutral kaon system at a $\phi$-factory, Nucl. Phys. B 868 (2013) 102 [arXiv:1208.0773 [hep-ph]].

[44] J. Bernabeu, A. Di Domenico and P. Villanueva-Perez, Probing CPT in transitions with entangled neutral kaons, JHEP 1510 (2015) 139 [arXiv:1509.02000 [hep-ph]].

[45] A. Gajos, Investigations of fundamental symmetries with the electron-positron systems, $\mathrm{PhD}$ thesis, Jagiellonian University (2018) [arXiv:1805.06009 [hep-ex]]. 